\title{
Solar System Observations with Spitzer Space Telescope: Preliminary Results
}

\author{
Dale P. Cruikshank ${ }^{\mathrm{a}}$ \\ ${ }^{a}$ NASA Ames Research Center, MS 245-6, Moffett Field, CA 94035-1000, USA \\ [Dale.P.Cruikshank@nasa.gov]
}

\begin{abstract}
The programs of observations of Solar System bodies conducted in the first year of the operation of the Spitzer Space Telescope as part of the Guaranteed Observing Time allocations are described. Initial results include the determination of the albedos of a number of Kuiper Belt objects and Centaurs from observations of their flux densities at 24 and $70 \mu \mathrm{m}$, and the detection of emission bands in the spectra of several distant asteroids (Trojans) around 10 and $25 \mu \mathrm{m}$. The 10 Kuiper Belt objects observed to date have albedos in the range $0.08-0.15$, significantly higher than the earlier estimated 0.04 . An additional $\mathrm{KBO}\left[(55565) 2002 \mathrm{AW}_{197}\right]$ has an albedo of $0.17 \pm 0.03$. The emission bands in the asteroid spectra are indicative of silicates, but specific minerals have not yet been identified. The Centaur/comet 29P/Schwassmann-Wachmann 1 has a nucleus surface albedo of $0.025 \pm 0.01$, and its dust production rate was calculated from the properties of the coma. Several other investigations are in progress as the incoming data are processed and analyzed.
\end{abstract}

\section{Keywords:}

\section{Introduction}

The Spitzer Space Telescope, initially known as SIRTF, is an $85-\mathrm{cm}$ diameter cryogenic telescope operating in a heliocentric orbit. The telescope, spacecraft, and the three principal instruments (MIPS, IRS, and IRAC) have been described in detail in Werner et al. (2004), Houck et al. (2004), Fazio et al. (2004), and Rieke et al. (2004). Spitzer has the operational capability to find and track Solar System bodies when they lie in a band of the sky defined by a minimum elongation of $85^{\circ}$ and a maximum elongation of $120^{\circ}$ from the Sun. This means that these objects cannot be observed near or at opposition, but distant Solar System objects can be observed for two periods of about 45 days each year. Objects closer to the Sun in highly elliptical orbits (e.g., comets and certain asteroids) are visible at less regular intervals and for variable periods of time.

As a part of the program of observations conducted by Spitzer scientists having guaranteed observing time (GTO), a large number of Solar System objects were included for spectroscopy $(5-40 \mu \mathrm{m})$ and imaging at several wavelengths in the range 3.6-160 $\mu \mathrm{m}$. Additional Solar System studies were conducted in the program of early release observations undertaken in the first months of operation of Spitzer. Approximately one year has elapsed since Spitzer went into full science operational mode, and we can at this 
point summarize some of the preliminary results of the programs of Solar System observations.

\section{Solar System Programs}

Planets: All of the planets through Satum are too bright to be observed by most instruments on Spitzer. Observations of Uranus, Neptune, and Pluto are included in the programs of spectroscopy and (for Pluto) photometric and radiometric imaging. The radiometric program is focused on determining the thermal inertia of Pluto's surface by observations over a range of longitudes as the planet rotates. These observations have been acquired but not yet evaluated. Spectroscopy of Pluto can only be accomplished for wavelengths $\lambda>15 \mu \mathrm{m}$; some data have been obtained but are not yet evaluated. Additional photometric measurements of Pluto with IRAC at 3.6, 4.5, 5.0, and $8.0 \mu \mathrm{m}$ have also been obtained for six equally spaced longitudes on the planet's surface. In all studies of Pluto, the flux from its satellite is also included; Spitzer does not have sufficient angular resolution to separate the planet from the satellite. Spectra of Neptune and Uranus have also been obtained and are being studied.

Planetary Satellites: Several large satellites of the outer planets are sufficiently separated from their planet in an angular sense to permit observations with various instruments on Spitzer, and some observations have already been conducted; the data are under evaluation.

Comets from Cradle to Grave: Approximately half of the comets entering the planetary region of the Solar System originate in the trans-Neptunian region called the Kuiper Belt. The Centaur objects orbiting the Sun beyond Jupiter and crossing the orbits of one or more major planets are in some sense an intermediate class between Kuiper Belt objects (KBOs) and traditional active short-period comets. Some Centaurs show episodic cometary activity and others do not. The properties and behaviors of ordinary comets are well known, but when comets have lost their volatile materials, they can in some instances be mistaken for asteroids. The study of the physical properties (dimensions, surface albedos, compositions, thermal properties) of all of the objects in the continuum from $\mathrm{KBO}$ to Centaurs to active comets to extinct comets can reveal important information the reflects on the basic nature of the condensation of solid material in the outer solar nebula and the subsequent evolution of the objects accreted in the earliest times of the existence of the Solar System.

Thus, the Spitzer early programs include spectroscopy, photometry, and radiometry of Kuiper Belt objects, Centaurs, traditional comets, and asteroidal objects suspected of being extinct comets on the basis of their orbital parameters.

Asteroids and the Source of the Carbonaceous Meteorites: The mineralogy of asteroids is known primarily from reflectance spectroscopy in the visible and nearinfrared spectral regions (e.g., Gaffey et al. 2002), and in the mid-infrared (e.g., Cohen et al. 1998, Dotto et al. 2002). Spectra in these regions are primarily diagnostic of Ca-, Fe-, and $\mathrm{Mg}$-bearing silicates, usually in olivines or pyroxenes. While this accounts for many 
of the minerals in chondritic and certain achondritic meteorites, the information is not diagnostic for identifying asteroid sources of the carbonaceous meteorites. The parent body or bodies of the carbonaceous meteorites are commonly thought to be one or more of the low-albedo, black or red-colored asteroids (classes C, P, and D, as well as subclasses), but in the absence of diagnostic spectral features a direct connection has not been reliably established. One component of the Spitzer asteroid program has been to search for diagnostic spectral features that will bear upon the question of the parent bodies of the carbonaceous meteorites by extending the wavelength region and the signal precision beyond that previously achieved, in search of diagnostic spectral features. Accordingly, a number of $\mathrm{C}, \mathrm{P}$, and $\mathrm{D}$ class asteroids are included in the initial target selection; spectra are currently being received and evaluated.

The Spitzer asteroid program also includes a spectroscopic study of objects that have been visited by spacecraft (253 Mathilde, 243 Ida, 951 Gaspra and 433 Eros) for which more information is available on surface composition and microstructure, size, shape, density, etc. Spitzer spectra for these for objects have been obtained and are under study.

Another component of the asteroid program is a spectroscopic study of the brightest members of the Trojan dynamical class. These objects tend to have very low albedos and red colors, and have long been thought to have complex organic materials on their surfaces (e.g., Gradie and Veverka 1980). More recent investigations suggest that their colors and albedos might result from mafic minerals on their surfaces (Cruikshank et al, 2001; Emery and Brown 2004), but a more definitive identification of surface materials is desired. The origin of the Trojans is in question; they may have been asteroids that accreted between Mars and Jupiter in the silicate-rich region of the solar nebula, or they may have condensed at larger heliocentric distances where ices were abundant, and subsequently captured into stable configurations in Jupiter's orbit. We are searching for spectroscopic and other evidence of the source(s) of the Trojan asteroids. Data have been obtained and analyzed for a few Trojan asteroids and are discussed below.

\section{Summary of Results So Far Obtained}

Kuiper Belt Objects and Centaurs: Several KBOs and Centaurs have been observed spectroscopically and radiometrically. Here we focus on the early results from the radiometric measurements at 24 and $70 \mu \mathrm{m}$ with the Spitzer MIPS instrument. Flux density measurements at two thermal wavelengths and one measurement of the reflected sunlight (at visible wavelengths) permit a determination of the size and albedo of a solid body. When additional information on the shape, rotation period and pole orientation are available (from ground-based telescopic data), the thermal inertia of the surface layers of KBOs and Centaurs can be calculated using modeling techniques that have been perfected and calibrated with observations of asteroids.

Thermal radiation from Kuiper Belt object (55565) $2002 \mathrm{AW}_{197}$ has been observed at 24 and $70 \mu \mathrm{m}$ and analyzed with models, with the result that the geometric albedo is $0.17 \pm 0.03$, a value that is much larger than that of comet nuclei (typically 
0.04) (Cruikshank et al. 2005). The discovery of the unexpectedly high albedo of this particular object, plus the trend of albedos of about 10 other KBOs to lie in the range 0.08 -0.15 , is changing the view in place for a decade of discoveries and studies of KBOs and related trans-neptunian bodies that these objects have comet-like albedos of order 0.04 . A further consequence of the measurement of higher albedos than expected is that these objects are smaller than originally estimated; the diameter of $2002 \mathrm{AW}_{197}$ is found to be $700 \pm 50 \mathrm{~km}$, and the sizes of KBOs may be typically only $60 \%$ as large as originally estimated.

The study of $2002 \mathrm{AW}_{197}$ also showed that neither of two widely used thermal models (the Standard Thermal Model and the Isothermal Latitude Model) accurately predicts the flux densities observed in the Cruikshank et al. (2005) study. The surface layers of this body must, therefore, have a small, but not negligible thermal inertia, which is a parameter that indicates the rate at which a body's temperature responds to changing heat input (insolation). Thermal inertia is proportional to the square root of the product of thermal conductivity and volume heat capacity.

Several Centaurs have also been observed at 24 and $70 \mu \mathrm{m}$, with the result that their albedos are even more diverse (and generally lower) than those of the KBOs, lying in the range $0.02-0.12$. While the Standard Thermal Model (STM) (Lebofsky and Spencer 1989) fits the flux densities observed for Centaurs better than for KBOs, these bodies also appear to have significant thermal inertias.

Several KBOs and Centaurs have also been observed spectroscopically; their reflected radiation is below the detection threshold of Spitzer's spectrometer, and their thermal flux densities can be observed reliably only at wavelengths $>10$ or $15 \mu \mathrm{m}$. Some spectral structure is detected in a few of these objects, perhaps indicative of fine-grained silicates in their surface materials, but the analysis is in a very preliminary state.

Mineralogy of Asteroids: To date, about 16 asteroids have been observed spectroscopically (Emery et al. 2005) in the Spitzer program, with good data acquired in the region $8-36 \mu \mathrm{m}$. The analysis has focused primarily on the objects showing the greatest spectral contrast, and this turns out to be three Trojans (624 Hektor, 911 Agamemnon, and 1172 Aneas). In order to study individual emission features in these thermal-region spectra, it is first necessary to remove the continuum emission reliably; this must be done with a rigorous model that accounts for the overall spectral energy distribution. The simplest approach is to use the Standard Thermal Model noted above. This model assumes the ideal situation of a non-rotating spherical body in instantaneous equilibrium with insolation, and also assumes that the subsolar and sub-observer points on the body coincide. The observed thermal emission is then a function only of the distances to the Sun and the observer, and the dimensions of the body. In the analysis of the Spitzer thermal spectra, the spectral energy distribution is calculated with the STM and the IAU absolute magnitude $(\mathrm{H})$ and slope $(\mathrm{G})$ parameters for the relationship between size and albedo in the visible spectral region. Leaving the radius and albedo as free parameters, the STM that best fits the energy distribution is found. The resulting diameter and albedo can be compared with independently determined values for 
verification. After the overall spectral energy distribution spectrum is removed from the data, the residuals represent the emission of specific classes of minerals and (potentially) other components such as ices and solid organic materials.

The early analysis of the asteroid spectra obtained to date shows that there is a variety of emission structures. In general terms, the contrast or strength of these emission features above the continuum is greater for distant asteroids (the Trojans) than for objects in the asteroid Main Belt. In the specific case of the three Trojans identified above, there is an emission plateau from $-9-15 \mu \mathrm{m}$ with a spectral contrast in excess of $10 \%$, and a broad peak centered near $20 \mu \mathrm{m}$. The spectra rise from $\sim 30 \mu \mathrm{m}$ to the end of the spectral range. the plateau around $10 \mu \mathrm{m}$ is identified as the Si-O stretching fundamental and that at $20 \mu \mathrm{m}$ is the $\mathrm{Si}-\mathrm{O}$ bending fundamental in silicate minerals. These emissions are seen at-these wavelengths only when the grain size is very small (few $\mu \mathrm{m}$ ), and are similar to the silicate emissions detected in comets (e.g., Wooden et al. 2004), although apparently showing fewer details that would be diagnostic of crystalline versus amorphous phases of the minerals.

Centaur/Comet 29P/Schwassmann-Wachmann 1: Spitzer obtained thermal images and spectra of comet and Centaur object 29P/Schwassmann-Wachmann 1 in late 2003 November as part of the early release observations with Spitzer (Stansberry et al. 2004). Images at 8,24 , and $70 \mu \mathrm{m}$ revealed an extensive coma, which at $24 \mu \mathrm{m}$ extended at least $8^{\prime}$ from the nucleus and showed a single jet. The dust production rate was estimated as $<\sim 50 \mathrm{~kg} \mathrm{~s}^{-1}$. The 24 to $70 \mu \mathrm{m}$ color temperature of the coma was $160 \mathrm{~K}$. The debris trail was also detected at $24 \mu \mathrm{m}$ with an optical depth of $\sim(7+/-3) \times 10^{-9}$. Thermal models fitted to photometry at 8,24 , and $70 \mu \mathrm{m}$ give a radius of $27+1-5 \mathrm{~km}$ for the nucleus, larger than all previous size estimates, and a geometric albedo of $0.025+/-0.01$, lower than any other Centaur object, but consistent with other comets. The spectra reveal features at 11.3 and $34 \mu \mathrm{m}$, which are tentatively identified as emission from the $\mathrm{Mg}$-rich olivine forsterite. This is the first identification of the mineralogy of the dust emitted by a Centaur object.

Comets: A number of other comets have been observed as a part of a separate program, and the results summarized by Gehrz et al. (2005).

\section{Conclusion}

In addition to the on-going programs of Solar System observations undertaken as part of the Guaranteed Time Observing, projects approved in the Guest Observer program are in progress. Spitzer promises to yield a wealth of unique information about Solar System bodies because of the high sensitivity of its three instruments and its expected long mission lifetime.

\section{Acknowledgements}

The initial programs of Solar System studies with Spitzer reported here have been undertaken by the author in collaboration with John A. Stansberry, George H. Rieke, 
James A. Houck, Michael W. Werner, Joshua P. Emery, Yanga R. Fernández, Jeffrey Van Cleve, William T. Reach, and Victoria S. Meadows. We thank the long-enduring Science Working Group and SIRTF/Spitzer Project, as well as the Spitzer operations staff, instrument teams, and others who have contributed to the success of this project.

\section{References}

Cohen, M., Witteborn, F. C., Roush, T., et al. Spectral irradiance calibration in the infrared VII. 5-14 micron spectroscopy of the asteroids Ceres, Vesta, and Pallas. Astron. J. 115, 1671-1679, 1998

Cruikshank, D. P., Dalle Ore, C. M., Roush, T. L., et al. Constraints on the composition of Trojan asteroid 624 Hektor, Icarus 153, 348-360 2001

Cruikshank, D. P., Stansberry, J. A., Emery, J. P., et al. The High-Albedo Kuiper Belt Object (55565) 2002 AW197. Astrophys. J. Lett. 2005 ( submitted)

Dotto, E., Barucci, M. A., and Müller, T. G., ISO observations of low and moderate albedo asteroids. PHT-P and PHT-S results. Astron. \& Astrophys. 393, 1065-1072, 2002

Emery, J. P., and Brown, R. H. The surface composition of Trojan asteroids: constraints set by scattering theory. Icarus $170,131-152,2004$

Emery, J. P., Cruikshank, D. P., Van Cleve, J., and Stansberry, J. A. Mineralogy of asteroids from observations with the Spitzer Space Telescope. LPSC 37, CD-ROM (abstract) 2005

Fazio, G. G., Hora, J. L., Allen, L. E., et al. The Infrared Array Camera (IRAC) for the Spitzer Space Telescope, Astrophys. Supp. Series, 10-15, 2004

Gaffey, M. J., Cloutis, E. A., Kelley, M. S., and Reed, K. L. Mineralogy of asteroids, in: Asteroids III, Bottke, W. F. Jr. et al. (eds), U. Arizona Press, pp. 183-204, 2002

Gehrz, R. D. et al. Infrared observations of comets with the Spitzer Space Telescope, 2005 , this volume

Gradie, J., and Veverka, J. The composition of the Trojan asteroids, Nature 283, 840-842, 1980

Houck, J. R., Roellig. T. L. van Cleve, J. The infrared spectrograph (IRS) on the Spitzer Space Telescope, Astrophys. J. Supp. Series 154, 18-24, 2004

Lebofsky, L. A., and Spencer, J. R. Radiometry and thermal modeling of asteroids, in Asteroids II, Binzel, R. P. et al, (eds), U. Arizona Press, pp. 128-136 
Rieke, G. H., Young, E. T., Engelbracht, C. W., et al. The multiband imaging photometer for Spitzer (MIPS), Astrophys. J. Supp. Series 154, 25-29

Stansberry, J. A., Van Cleve, J., Reach, W. T et al. Spitzer observations of the dust coma and nucleus of 29P/Schwassmann-Wachmann 1. Astrophys. J. Supp. Series 154, 463468,2004

Werner, M. W., Roellig, T. L., Low, F. J., et al. The Spitzer Space Telescope Mission, . Astrophys. J. Supp. Series 154, 1-9, 2004

Wooden, D. H., Woodward, C. E., and Harker, D. E. Discovery of crystalline silicates in comet C/2001 Q4 (NEAT), Astrophys. J. Lett. L77-L80, 2004 